\title{
The join of the pseudovarieties of idempotent semigroups and locally trivial semigroups
}

\author{
M. Zeitoun ${ }^{1}$ \\ Communicated by J.E. Pin
}

\begin{abstract}
This article solves a problem proposed by Almeida: the computation of the join of two well-known pseudovarieties of semigroups, namely the pseudovariety of bands and the pseudovariety of locally trivial semigroups. We use a method developed by Almeida, based on the theory of implicit operations.
\end{abstract}

\section{Introduction}

The problem solved in this paper was originally motivated by the classification of rational languages.

The description of the lattice of varieties of semigroups is a famous problem in semigroup theory. Important results in this direction were given by Biryukov [15], Fennemore [18, 19] and Gerhard [20], who described the lattice of varieties of bands, and by Polák [26, 27, 28] who described the lattice of varieties of completely regular semigroups.

There is an analogous problem for pseudovarieties, which is mainly motivated by considerations of automata theory. Indeed, Eilenberg's variety theorem [31] gives a bijective correspondence between varieties of rational languages and pseudovarieties of semigroups. In particular, the join $\mathbf{V} \vee \mathbf{W}$ of two pseudovarieties of semigroups $\mathbf{V}$ and $\mathbf{W}$ corresponds to the smallest variety of languages containing the varieties corresponding to $\mathbf{V}$ and $\mathbf{W}$.

Little is known on the join of two pseudovarieties, even when they are simple. In contrast, its definition is straightforward, and it appears in a very natural way, when considering parallel computations of automata.

Most results concerning this problem are recent. Several kinds of partial or particular answers were given:

- Some computations can be done explicitly. The main technique to compute a join is to use the theory of implicit operations. This theory was developed and used in numerous applications by Almeida [7, 4]. Reiterman's theorem [29] is the starting point of the equational theory of pseudovarieties: it states that pseudovarieties are defined by pseudoidentities, just as varieties are defined by identities. Using this

\footnotetext{
${ }^{1}$ This work was partly supported by PRC Mathématiques et Informatique and by ESPRITBRA WG 6317 ASMICS-2
} 
theorem and some ad hoc facts, Almeida, Azevedo and Weil [3, 2, 8, 10, 11, 14, 13] computed some non-trivial joins for which algebraic methods failed.

- Sometimes, one can only determine whether the join has a finite basis of pseudoidentities. For instance, Almeida [6] proved that the commutative pseudovarieties are finitely based, while Volkov [33], Trotter and Volkov [32] and the author [35] gave examples of non finitely based joins.

- In most cases, however, nothing can be proved about the basis describing the pseudovariety. The main problem is then the decidability of the pseudovariety $\mathbf{V}$ : given a finite semigroup $S$, is the membership of $S$ in $\mathbf{V}$ decidable? Once again, the problem is very difficult. A recent result of Albert, Baldinger and Rhodes [1] states that even the join of two decidable pseudovarieties might not be decidable.

Rhodes [30], Almeida [7] and Kharlampovich and Sapir [23] proposed a list of problems on pseudovarieties, most of which are still open. In this article, we solve problem $\mathbf{2 3}$ of Almeida [7]: "Compute $\mathbf{L I} \vee \mathbf{B}$ ", where $\mathbf{L I}$ is the pseudovariety of semigroups whose local subsemigroups are trivial, and $\mathbf{B}$ is the pseudovariety of bands. We give a basis and a simple algebraic characterization for this join. We also give a description of the implicit operations on $\mathbf{L I} \vee \mathbf{B}$.

The paper is organized as follows: after a brief section on basic definitions, the main result: the computation of $\mathbf{L I} \vee \mathbf{B}$ (Theorem 3.1 below) is stated and proved in section 3. Section 4 is devoted to the computation of some subpseudovarieties of $\mathbf{L I} \vee \mathbf{B}$. Finally, in section 5, we analyse the structure of the implicit operations on $\mathbf{L I} \vee \mathbf{B}$.

\section{Notations and background}

We briefly review the main definitions and some useful facts about semigroups, pseudovarieties and implicit operations. For more details, the reader is referred to the books of Almeida [7], Pin [25], to the original article of Almeida [4] or for a brief introduction, to the surveys of Almeida and Weil [5, 10, 11, 34]. We assume the reader to be familiar with some basic notions of topology, universal algebra and semigroup theory (see for instance [16, 17, 25]).

\section{1.. Semigroups and pseudovarieties}

In the sequel, $A_{n}$ denotes the finite alphabet $\left\{x_{1}, \ldots, x_{n}\right\}$. For a word $u \in A_{n}^{+}$, the content $c(u)$ of $u$ is the set of all letters appearing in $u$. The length $|u|$ of $u$ is the number of letters of $u$.

The subset of idempotent elements of a semigroup $S$ is denoted by $E(S)$. If $S$ is finite, the exponent of $S$ is the least integer $k$ such that $s^{k}$ is idempotent for every $s \in S$. Recall that the local subsemigroup of $S$ associated to the idempotent $e$ is the subsemigroup $e S e$ of $S$.

A pseudovariety of semigroups is a class of finite semigroups closed under formation of finite direct product, homomorphic image and subsemigroup. Here is a list of some important pseudovarieties:

- The pseudovariety of all finite semigroups is denoted by $\mathbf{S}$.

- The pseudovariety of all finite aperiodic (or group-free) semigroups is denoted by 
A.

- An idempotent semigroup is called a band. The pseudovariety of finite bands is denoted by $\mathbf{B}$.

- The pseudovariety of finite commutative bands (that is, of finite semilattices) is denoted by $\mathbf{S l}$.

- LI denotes the pseudovariety of all semigroups whose local subsemigroups are trivial. It has some well-known subpseudovarieties:

- for every $k \in \mathbb{N}, \mathbf{L I}_{\mathbf{k}}$ is the pseudovariety of all finite semigroups satisfying the identity $x_{1} \ldots x_{k} y z_{1} \ldots z_{k}=x_{1} \ldots x_{k} z_{1} \ldots z_{k}$.

- K (resp. D) is the pseudovariety of all finite semigroups in which idempotents are left zeros (resp. right zeros).

- for every $k \in \mathbb{N}, \mathbf{K}_{\mathbf{k}}$ (resp. $\mathbf{D}_{\mathbf{k}}$ ) is the pseudovariety of all finite semigroups such that every product of $k$ elements of $S$ is a left zero (resp. a right zero).

- $\mathbf{N}$ is the pseudovariety of all finite nilpotent semigroups, that is, semigroups with a zero which is the unique idempotent.

- for every $k \in \mathbb{N}, \mathbf{N}_{\mathbf{k}}$ is the pseudovariety of all finite nilpotent semigroups $S$ such that every product of $k$ elements of $S$ is zero.

- Finally, for any pseudovariety $\mathbf{V}, \mathbf{D V}$ is the pseudovariety of all semigroups whose regular $\mathbf{D}$-classes are semigroups of $\mathbf{V}$. In particular, DS consists of all finite semigroups whose regular $\mathbf{D}$-classes are semigroups, and $\mathbf{D A}$ of all finite semigroups whose regular $\mathbf{D}$-classes are aperiodic semigroups.

It is important to keep in mind the fact that a pseudovariety containing $\mathbf{N}$, like $\mathbf{L I}$, cannot satisfy any non-trivial identity. Indeed, $\mathbf{N}_{\mathbf{k}}$ does not satisfy any non-trivial identity $u=v$ where $|u|<k$ and $|v|<k$. This simple property will be used several times in the sequel.

Recall that the free band over $A_{n}$ is a finite semigroup, quotient of the free semigroup $A_{n}^{+}$by the congruence generated by $x=x^{2}[22,24]$. We denote this congruence over $A_{n}^{+}$by $\equiv_{n}$.

A relational morphism $\tau$ from a semigroup $S$ to a semigroup $T$ is a relation that associates to each $s \in S$ a non empty subset $s \tau$ of $T$ such that

$$
\forall s, s^{\prime} \in S, \quad(s \tau)\left(s^{\prime} \tau\right) \subseteq\left(s s^{\prime}\right) \tau
$$

The Mal'cev product $\mathbf{V} m \mathbf{W}$ of two pseudovarieties $\mathbf{V}$ and $\mathbf{W}$ is the pseudovariety of all finite semigroups $S$ satisfying the following property: there exists a relational morphism $\tau$ from $S$ into a semigroup of $\mathbf{W}$ such that the inverse image of every idempotent of $S$ lies in $\mathbf{V}$.

\section{2.. Implicit operations}

Let $\mathbf{V}$ be a given pseudovariety. A semigroup $S$ separates two words $u$ and $v$ of $A_{n}^{+}$if there exists a semigroup morphism $\varphi: A_{n}^{+} \rightarrow S$ such that $u \varphi \neq v \varphi$. One defines a pseudo-metric $e_{\mathbf{v}}$ on $A_{n}^{+}$as follows:

$$
r_{\mathbf{v}}(u, v)=\min \{|S| \mid S \in \mathbf{V} \text { and separates } u \text { and } v\}
$$

and

$$
e_{\mathbf{v}}(u, v)=2^{-r_{\mathbf{v}}(u, v)}
$$


By convention, $\min \varnothing=\infty$ and $2^{-\infty}=0$.

Thus, $e_{\mathbf{v}}(u, v) \leq 2^{-k}$ if and only if $u$ and $v$ have the same evaluation on every semigroup of $\mathbf{V}$ whose size is less than $k$. It is not difficult to verify the following, for all $u, v, w \in A_{n}^{+}$:

1. $e_{\mathbf{v}}(u, u)=0$,

2. $e_{\mathbf{V}}(u, v)=e_{\mathbf{v}}(v, u)$,

3. $e_{\mathbf{v}}(u, v) \leq \max \left(e_{\mathbf{v}}(u, w), e_{\mathbf{v}}(w, v)\right)$,

4. $e_{\mathbf{V}}(u w, v w) \leq e_{\mathbf{V}}(u, v)$ and $e_{\mathbf{V}}(w u, w v) \leq e_{\mathbf{V}}(u, v)$.

It is then straightforward to see that the relation $\sim_{\mathbf{v}}$ defined by

$$
u \sim_{\mathbf{v}} v \Longleftrightarrow e_{\mathbf{V}}(u, v)=0
$$

is a congruence. The quotient $A_{n}^{+} / \sim_{\mathbf{v}}$ is a semigroup, denoted by $\mathrm{F}_{n}(\mathbf{V})$. In fact, $\mathrm{F}_{n}(\mathbf{V})$ is the free semigroup of the variety generated by $\mathbf{V}$. Furthermore, if $\mathbf{V}$ is not trivial, there exists in $\mathbf{V}$ a semigroup with at least two elements that separates $x_{i}$ and $x_{j}$ for $i \neq j$. Hence, the function $x_{i} \mapsto x_{i} / \sim_{\mathrm{v}}$ is a bijection. We assume now that $\mathbf{V}$ is not trivial and we identify $x_{i}$ with $x_{i} / \sim_{\mathbf{v}}$ and $A_{n}$ with $A_{n} / \sim_{\mathbf{v}}$.

By properties 1. to 3., $e_{\mathbf{V}}$ induces an ultrametric distance function $d_{\mathbf{v}}$ over $\mathrm{F}_{n}(\mathbf{V})$. By property 4., the multiplication in $\mathrm{F}_{n}(\mathbf{V})$ is uniformly continuous for this metric, so that $\mathrm{F}_{n}(\mathbf{V})$ is a topological semigroup. The completion of the metric space $\left(\mathrm{F}_{n}(\mathbf{V}), d_{\mathbf{V}}\right)$ is denoted by $\overline{\mathrm{F}}_{n}(\mathbf{V})$. Thus, $\overline{\mathrm{F}}_{n}(\mathbf{V})$ is a compact totally disconnected topological semigroup, and $\mathrm{F}_{n}(\mathbf{V})$ is dense in $\overline{\mathrm{F}}_{n}(\mathbf{V})$. The elements of $\overline{\mathrm{F}}_{n}(\mathbf{V})$ are called the $n$-ary implicit operations. Implicit operations that lie in fact in $\mathrm{F}_{n}(\mathbf{V})$ are said to be explicit.

When $\mathrm{F}_{n}(\mathbf{V})$ is finite, then $\overline{\mathrm{F}}_{n}(\mathbf{V})=\mathrm{F}_{n}(\mathbf{V})$. This happens for $\mathbf{V}=\mathbf{S l}$ and for $\mathbf{V}=\mathbf{B}$, for instance. $\mathrm{F}_{n}(\mathbf{S l})$ is the free semigroup of the variety of semilattices: it is isomorphic to the semigroup $\left(\mathbf{P}\left(A_{n}\right), \cup\right)$, where $\mathbf{P}\left(A_{n}\right)$ is the power set of $A_{n}$. Thus, a subset of $A_{n}$ determines a unique implicit operation on $\overline{\mathrm{F}}_{n}(\mathrm{Sl})$. For example, the operations $x_{4} x_{3}^{3} x_{1}$ and $x_{1} x_{3} x_{4}$ are equal because they have the same content: $\left\{x_{1}, x_{3}, x_{4}\right\}$.

Every explicit operation $u=u\left(x_{1}, \ldots, x_{n}\right) \in \mathrm{F}_{n}(\mathbf{V})$ defines for every semigroup $S \in \mathbf{V}$ a function $u_{S}$ from $S^{n}$ into $S$ : $u_{S}\left(s_{1}, \ldots, s_{n}\right)=u\left(s_{1}, \ldots, s_{n}\right)$. The function $u_{S}$ is called the evaluation of $u$ over $S$. For instance, the evaluation of $u=x_{1} x_{4}^{2} x_{3} \in \mathrm{F}_{4}(\mathbf{V})$ is the function $u_{S}$ from $S^{4}$ into $S$ such that $u_{S}(a, b, c, d)=a d^{2} c$. It is easy to see that one can define the evaluation $\pi_{S}$ of an implicit operation $\pi \in \overline{\mathrm{F}}_{n}(\mathbf{V})$ over a semigroup $S \in \mathbf{V}$ as follows: if $\left(u_{k}\right)_{k}$ is a sequence of explicit operations whose limit is $\pi$, then the sequence $\left(\left(u_{k}\right)_{S}\right)_{k}$ converges to a value that does not depend on the sequence $\left(u_{k}\right)_{k}$. By definition, $\pi_{S}$ is this limit value.

A pair $(\pi, \rho) \in \overline{\mathrm{F}}_{n}(\mathbf{V}) \times \overline{\mathrm{F}}_{n}(\mathbf{V})$ is called a pseudoidentity. Let $(\pi, \rho)$ be a pseudoidentity. A semigroup $S \in \mathbf{V}$ satisfies $(\pi, \rho)$ if and only if $\pi_{S}=\rho_{S}$. We 
will then write $S \models \pi=\rho$. If $\Sigma$ is a set of pseudoidentities on a pseudovariety $\mathbf{V}$, $S$ satisfies $\Sigma$ if $S$ satisfies every pseudoidentity of $\Sigma$, and a class $\mathbf{C}$ of semigroups satisfies $\Sigma$ if every semigroup of $\mathbf{C}$ satisfies $\Sigma$. We will then write $\mathbf{C} \models \Sigma$. The class $\llbracket \Sigma \rrbracket_{\mathbf{V}}$ of all semigroups of $\mathbf{V}$ satisfying $\Sigma$ is a pseudovariety. In the sequel, we shall write $\llbracket \Sigma \rrbracket$ instead of $\llbracket \Sigma \rrbracket_{\mathbf{S}}$.

The following proposition comes from the definitions and will be used many times:

Proposition 2.1. $\quad$ Let $\mathbf{V}$ be a pseudovariety, and $\left(u_{m}\right)_{m}$ a sequence of explicit operations of $\mathrm{F}_{n}(\mathbf{V})$. Then, this sequence converges if and only if

$$
\forall S \in \mathbf{V}, \exists m \in \mathbb{N}, \forall p, q \geq m, \quad\left(u_{p}\right)_{S}=\left(u_{q}\right)_{S}
$$

and it converges to an implicit operation $\pi$ if and only if

$$
\forall S \in \mathbf{V}, \exists m \in \mathbb{N}, \forall p \geq m, \quad\left(u_{p}\right)_{S}=\pi_{S}
$$

This proposition has an important consequence: given an implicit operation $\pi$, the sequence $\left(\pi^{k !}\right)_{k}$ converges. The limit of this sequence is denoted by $\pi^{\omega}$. For any letter $x$, the evaluation of $x^{\omega}$ on an element $s$ of a finite semigroup is the unique idempotent of the subsemigroup generated by $s$.

The following characterization of pseudovarieties is the fundamental theorem of the equational theory of pseudovarieties; notice the analogy with Birkhoff's completeness theorem:

Theorem 2.2. $\quad$ Reiterman [29] Let $\mathbf{V}$ be a pseudovariety of semigroups and let $\mathbf{W}$ be a subclass of $\mathbf{V}$. Then, $\mathbf{W}$ is a pseudovariety if and only if there exists a set of pseudoidentities $\Sigma \subseteq \overline{\mathrm{F}}_{n}(\mathbf{V}) \times \overline{\mathrm{F}}_{n}(\mathbf{V})$ such that $\mathbf{W}=\llbracket \Sigma \rrbracket_{\mathbf{V}}$.

For example, $\mathbf{B}$ is defined by the pseudoidentity $x=x^{2}$. In the same way, $\mathbf{S l}=\llbracket x=x^{2}, \quad x y=y x \rrbracket=\llbracket x y=y x \rrbracket_{\mathbf{B}}$. More generaly, $\mathbf{A}$ is equal to $\llbracket x^{\omega}=x^{\omega+1} \rrbracket$. Let us now see some other examples. It is straightforward to check that a semigroup belongs to $\mathbf{K}$ if and only if every idempotent is a left zero. Therefore, the pseudovariety $\mathbf{K}$ can be defined by the pseudoidentity $x^{\omega} y=x^{\omega}$. Similarly, $\mathbf{D}=\llbracket y x^{\omega}=x^{\omega} \rrbracket$.

Notice also that if $\mathbf{V}_{\mathbf{1}}$ (resp. $\mathbf{V}_{\mathbf{2}}$ ) is defined by $\Sigma_{1}$ (resp. $\Sigma_{2}$ ), then $\mathbf{V}_{\mathbf{1}} \cap \mathbf{V}_{\mathbf{2}}$ is defined by $\Sigma_{1} \cup \Sigma_{2}$. Thus, $\mathbf{N}=\mathbf{K} \cap \mathbf{D}$ is defined by $x^{\omega} y=x^{\omega}=y x^{\omega}$. For convenience, we will write $\pi=0$ instead of $\pi x_{i}=x_{i} \pi=0$ if $x_{i}$ is a letter. Thus, $\mathbf{N}=\llbracket x^{\omega}=0 \rrbracket$. This example shows that the computation of a basis of an intersection $\mathbf{V}_{\mathbf{1}} \cap \mathbf{V}_{\mathbf{2}}$ is straightforward when bases of $\mathbf{V}_{\mathbf{1}}$ and $\mathbf{V}_{\mathbf{2}}$ are known.

In contrast, it is difficult to find a basis for the join $\mathbf{V}_{\mathbf{1}} \vee \mathbf{V}_{\mathbf{2}}$. Indeed, the pseudoidentities satisfied by $\mathbf{V}_{\mathbf{1}} \vee \mathbf{V}_{\mathbf{2}}$ are exactly those satisfied by both $\mathbf{V}_{\mathbf{1}}$ and $\mathbf{V}_{\mathbf{2}}$. In general, this characterization does not give much information about the exact form of these pseudoidentities. However, for $\mathbf{L I}=\mathbf{K} \vee \mathbf{D}$, it is easy to get such a basis: LI is defined by $x^{\omega} y x^{\omega}=x^{\omega}$, or alternatively by $x^{\omega} y z^{\omega}=x^{\omega} z^{\omega}$. Indeed, to say that a semigroup $S$ satisfies $x^{\omega} y x^{\omega}=x^{\omega}$ means that for every $e \in E(S)$ 
and every $s \in S$, the elements ese and $e$ are equal: the local subsemigroup $e S e$ is trivial.

Some definitions on words can now be extended for explicit or implicit operations. For instance, let $\mathbf{V}$ be a pseudovariety containing $\mathbf{N}$. Since V does not satisfy any non-trivial identity, the length $|u|$ of any explicit operation $u$ can again be defined as the number of letters of $u$.

One can also define the content of an implicit operation. For instance, it is natural to say that the content of $x^{\omega} y t^{\omega}$ is $\{x, y, z\}$. Notice however that $x^{\omega} y t^{\omega}$ is equal to $x^{\omega} t^{\omega}$ on $\overline{\mathrm{F}}_{n}(\mathbf{L I})$. In fact, the content can be defined in a satisfactory way for pseudovarieties containing Sl.

Theorem 2.3. Almeida, Azevedo [7, 13] Let $\mathbf{V}$ be a pseudovariety containing Sl. Then, two words representing an explicit operation $u$ on $\mathrm{F}_{n}(\mathbf{V})$ have the same content. We denote this content by $c(u)$. Then, the function $c: \mathrm{F}_{n}(\mathbf{V}) \longrightarrow \mathrm{F}_{n}(\mathbf{S l})$ can be extended in a unique way in a continuous homomorphism from $\overline{\mathrm{F}}_{n}(\mathbf{V})$ into $\overline{\mathrm{F}}_{n}(\mathrm{Sl})$.

Thus, for such a pseudovariety, the content of $x^{\omega} y t^{\omega}$ is $\{x, y, z\}$.

Implicit operations are in general very difficult to handle. An important simplification has been discovered by Almeida and Azevedo: on DS, implicit operations have a nice factorization. The structure of the $\mathbf{J}$ classes of $\overline{\mathrm{F}}_{n}(\mathbf{D S})$ plays an important role. It is described in the next statement:

Theorem 2.4. Azevedo [13] Let $\pi$ and $\rho$ be two regular elements of $\overline{\mathrm{F}}_{n}(\mathbf{D S})$. Then, $\pi \mathbf{J} \rho \Longleftrightarrow c(\pi)=c(\rho)$. Furthermore, DS is the largest pseudovariety satisfying this property.

The fundamental theorem is the following:

Theorem 2.5. Almeida, Azevedo [7, 9, 13, 12] Every implicit operation $\pi \in \overline{\mathrm{F}}_{n}(\mathbf{S})$ admits a factorization of the form $\pi=\pi_{1} \ldots \pi_{k}$ where $\pi_{i}$ is either explicit or its restriction to DS is regular.

Most non trivial computations of pseudovarieties obtained using the theory of implicit operations were based on this theorem. See [7] for examples.

\section{The pseudovariety $L I \vee B$}

This section is devoted to the proof of the main theorem:

Theorem 3.1. $\quad$ The pseudovariety $\mathbf{L I} \vee \mathbf{B}$ is determined by

$$
\mathbf{L I} \vee \mathbf{B}=\llbracket\left(x^{\omega} y\right)=\left(x^{\omega} y\right)^{2}, \quad\left(y x^{\omega}\right)=\left(y x^{\omega}\right)^{2} \rrbracket=\mathbf{B} m \mathbf{N} \cap \llbracket x^{\omega} y z^{\omega}=x^{\omega} y^{2} z^{\omega} \rrbracket
$$


This statement consists of two equalities. The second one is easier, and will be proved in Lemma 3.2. For the first equality, the inclusion from left to right is easy: notice that if $S$ is a semigroup that belongs to $\mathbf{L I}$ or $\mathbf{B}$, the set $E(S)$ of idempotents of $S$ is an ideal. Therefore, the pseudovariety $\llbracket\left(x^{\omega} y\right)=\left(x^{\omega} y\right)^{2}, \quad\left(y x^{\omega}\right)=$ $\left(y x^{\omega}\right)^{2} \rrbracket$ is a natural upper bound for $\mathbf{L I} \vee \mathbf{B}$. The proof of the opposite inclusion is based on Reiterman's theorem.

Let us now show the second equality of the theorem:

Lemma 3.2. The pseudovariety $\llbracket\left(x^{\omega} y\right)=\left(x^{\omega} y\right)^{2}, \quad\left(y x^{\omega}\right)=\left(y x^{\omega}\right)^{2} \rrbracket$ is equal to the Mal'cev product $\mathbf{B}$ (m) $\mathbf{N}$.

Proof. Let $S$ be a semigroup and assume that $E(S)$ is an ideal. Then, $S / E(S)$ is nilpotent and the canonical morphism $\tau: S \longrightarrow S / E(S)$ satisfies $\tau^{-1}(0) \in \mathbf{B}$. Conversely, if $S$ is in $\mathbf{B}: \mathbf{N}$, there exists a nilpotent semigroup $N$ of exponent $p$ and a relational morphism $\tau$ from $S$ into $N$ such that $\tau^{-1}(0)$ is a band. Pick then $e \in E(S)$ and $s \in S$. Since $\tau(e x)=\tau\left(e^{p} x\right) \supseteq \tau(e)^{p} \tau(x)=\{0\}$, one gets $e x \in \tau^{-1}(0)$, and therefore $e x$ is idempotent. One would show in the same way that $x e$ is also idempotent.

Next, since $\mathbf{L I}$ and $\mathbf{B}$ are in $\mathbf{D A}$, we shall check that $\mathbf{B} m \mathbf{N} \subseteq \mathbf{D A}$. This fact will be used later to apply the factorization theorem on DS.

Lemma 3.3. $\quad$ The pseudovariety $\mathbf{B} \rightarrow \mathbf{N}$ is included in $\mathbf{D A}$.

Proof. Let $S$ be a semigroup of $\mathbf{B} m \mathbf{N}$. We have to show that every regular D-class of $S$ is an aperiodic semigroup. In fact, $S$ satisfies itself $x^{\omega}=x^{\omega+1}$ : it suffices to take $x=y$ in one of the pseudoidentities defining $\mathbf{B}(m \mathbf{N}$.

Next, if $z$ lies in a regular $\mathbf{D}$-class, then there exists an idempotent $e$ such that $e \mathbf{R} z$, and therefore $e z=z$. Since $E(S)$ is an ideal, $z$ is itself an idempotent. Hence, for any $y$ such that $x \mathbf{D} y$, the $\mathbf{H}$-class $R_{y} \cap L_{x}$ contains an idempotent; therefore, $x y \in R_{x} \cap L_{y}$, and $x y$ lies in the same $\mathbf{D}$-class. Therefore, every regular D-class is a semigroup.

Remark 3.4. The inclusion $\mathbf{B} m \mathbf{N} \subseteq \mathbf{D A}$ is strict, otherwise $\mathbf{B} m \mathbf{N}$ would contain for instance the pseudovariety $\mathbf{J}$ of $\mathbf{J}$-trivial semigroups, but $\mathbf{J}$ does not satisfy the pseudoidentity $\left(x^{\omega} y\right)^{2}=x^{\omega} y$ (see Almeida [7]).

The rest of the proof follows the method developed by Almeida: Reiterman's theorem states that one can write $\mathbf{L I} \vee \mathbf{B}=\llbracket \Sigma \rrbracket_{\mathbf{B} \circledast \mathbf{N}}$, where $\Sigma$ is a set of pseudoidentities on $\mathbf{B}\left(m \mathbf{N}\right.$. This implies in particular that if $\left(\pi, \pi^{\prime}\right) \in \Sigma^{2}$, then both $\mathbf{L I}$ and $\mathbf{B}$ satisfy $\pi=\pi^{\prime}$. All we have to show now is that $\pi$ and $\pi^{\prime}$ are equal in $\overline{\mathrm{F}}_{n}(\mathbf{B}$ (m) $\mathbf{N})$.

Therefore, we need some information on the implicit operations on $\mathbf{B} m \mathbf{N}$. They take on a very particular form, as shown in the next proposition: 
Proposition 3.5. $\quad$ Let $\mathbf{V}$ be a pseudovariety such that $\mathbf{N} \subseteq \mathbf{V} \subseteq \mathbf{B} m \mathbf{N}$. The three following facts hold in $\overline{\mathrm{F}}_{n}(\mathbf{V})$ :

1. Every implicit operation of $\overline{\mathrm{F}}_{n}(\mathbf{V})$ is either explicit or idempotent.

2. An implicit operation of $\overline{\mathrm{F}}_{n}(\mathbf{V})$ cannot be both explicit and idempotent.

3. If $\left(u_{m}\right)_{m}$ is a sequence of explicit operations such that $\lim _{m \rightarrow \infty}\left|u_{m}\right|=+\infty$, then $\left(u_{m}\right)_{m}$ cannot converge to an explicit operation.

Proof. If $\pi$ is a regular implicit operation, then $\pi$ is idempotent by aperiodicity. Therefore, as $\mathbf{B}: \mathbf{N} \subseteq \mathbf{D A} \subseteq \mathbf{D S} \cap \mathbf{A}$, every implicit operation of $\overline{\mathrm{F}}_{n}(\mathbf{B}: \mathbf{N})$ has a factorization in a product of explicit and idempotent operations by Theorem 2.5. But $E\left(\overline{\mathrm{F}}_{n}(\mathbf{B}(\mathrm{m}) \mathbf{N})\right)$ is an ideal. Hence, such a product is idempotent as soon as it contains one idempotent operation. This proves 1 .

In order to prove 2, notice that if $u \in \mathrm{F}_{n}(\mathbf{V})$ is an idempotent, then $\mathbf{N} \subseteq \mathbf{V}$ would satisfy $u=u^{2}$. But $\mathbf{N}$ does not satisfy any non-trivial identity, so the words $u$ and $u^{2}$ are identical, which is impossible.

Finally, for 3, assume that a sequence $\left(u_{m}\right)_{m}$ of explicit operations converges to an explicit operation $u$, and let $k=|u|+1$. Since $\mathbf{N}_{\mathbf{k}} \subseteq \mathbf{V}$ and since $\mathrm{F}_{n}\left(\mathbf{N}_{\mathbf{k}}\right)$ is finite, $\overline{\mathbf{F}}_{n}\left(\mathbf{N}_{\mathbf{k}}\right)$ is a semigroup of $\mathbf{V}$. Since $u$ is the limit of $\left(u_{m}\right), \mathbf{N}_{\mathbf{k}}$ satisfies $u_{m}=u$ for $m$ large enough. This is in contradiction with the fact that $\mathbf{N}_{\mathbf{k}}$ does not satisfy any non-trivial identity $v=u$ where $|u|<k$ and $|v|<k$. So the limit of $\left(u_{m}\right)$ cannot be explicit.

Our next lemma deals with the pseudoidentity $x^{\omega} y x^{\omega}=x^{\omega} y^{2} x^{\omega}$. It is immediate that this pseudoidentity is satisfied by $\mathbf{L I}$ and by $\mathbf{B}$, and hence it is satisfied by $\mathbf{L I} \vee \mathbf{B}$. We show that it holds in $\mathbf{B}(m) \mathbf{N}$ too.

Lemma 3.6. $\quad$ The pseudovariety $\mathbf{B}: \mathbf{N}$ satisfies $x^{\omega} y x^{\omega}=x^{\omega} y^{2} x^{\omega}$.

Proof. Let $\pi=x^{\omega} y x^{\omega}$ and $\rho=x^{\omega} y^{2} x^{\omega}$ in $\overline{\mathrm{F}}_{n}(\mathbf{B}: \mathbf{N})$. By Lemma 3.2, these implicit operations are both idempotent. Furthermore, $\mathbf{S l} \subseteq \mathbf{B} m \mathbf{N} \subseteq \mathbf{D S}$. As $c(\pi)=c(\rho), \pi$ and $\rho$ lie in the same $\mathbf{J}$-class, by Theorem 2.4. By aperiodicity, it suffices therefore to show that they lie in the same $\mathbf{H}$-class. But

$$
x^{\omega} y x^{\omega} \cdot x^{\omega} y^{2} x^{\omega}=\left(x^{\omega} y\right)^{2} y x^{\omega}=x^{\omega} y^{2} x^{\omega}
$$

Similarly,

$$
x^{\omega} y^{2} x^{\omega} \cdot x^{\omega} y x^{\omega}=x^{\omega} y\left(y x^{\omega}\right)^{2}=x^{\omega} y^{2} x^{\omega}
$$

Therefore, $\pi \rho=\rho \pi=\rho$, and $\pi$ and $\rho$ are comparable for the relations $\mathbf{R}$ and $\mathbf{L}$. Since they lie in the same $\mathbf{J}$-class, they have to be $\mathbf{H}$-equivalent.

However, the next statement shows that one cannot simplify the intersection in Theorem 3.1. Indeed, $\mathbf{L I} \vee \mathbf{B}$ obviously satisfies the pseudoidentity $x^{\omega} y z^{\omega}=$ $x^{\omega} y^{2} z^{\omega}$ which fails in $\mathbf{B}$ : $\mathbf{N}=\llbracket\left(x^{\omega} y\right)=\left(x^{\omega} y\right)^{2}, \quad\left(y x^{\omega}\right)=\left(y x^{\omega}\right)^{2} \rrbracket$. 
Lemma 3.7. $\quad$ The pseudovariety $\mathbf{B}(m) \mathbf{N}$ does not satisfy the pseudoidentity $x^{\omega} y z^{\omega}=x^{\omega} y^{2} z^{\omega}$.

Proof. Consider the free 3-generated semigroup $\mathrm{F}$ in the semigroup variety defined by $x y=(x y)^{2}$. Gerhard proved in [21] that it is finite; therefore, it clearly lies in $\mathbf{B}(m \mathbf{N}$. The word problem in $\mathrm{F}$ was also solved in [21]. Gerhard's algorithm easily shows that the words $x^{2} y z^{2}$ and $x^{2} y^{2} z^{2}$ are different in $\mathrm{F}$.

Let now $\mathbf{U}=\mathbf{B}\left(m \mathbf{N} \cap \llbracket x^{\omega} y z^{\omega}=x^{\omega} y^{2} z^{\omega} \rrbracket\right.$ and $\left(\pi, \pi^{\prime}\right) \in\left(\overline{\mathrm{F}}_{n}(\mathbf{U})\right)^{2}$ be a nontrivial pseudoidentity satisfied by $\mathbf{L I} \vee \mathbf{B}$ : that is, $\pi \neq \pi^{\prime}$ and $\mathbf{L I} \vee \mathbf{B} \models \pi=\pi^{\prime}$. By Proposition 3.5, the operations $\pi$ and $\pi^{\prime}$ are either explicit or idempotent. Since in particular $\mathbf{N} \models \pi=\pi^{\prime}$ (since $\mathbf{N} \subseteq \mathbf{L I}$ ), and since $\mathbf{N}$ does not satisfy any non-trivial identity, $\pi$ and $\pi^{\prime}$ are not both explicit. For the same reason, if $\pi^{\prime}$ is idempotent, then so is $\pi$ : indeed, if $\pi^{\prime}$ was idempotent and $\pi$ explicit, we would have $\mathbf{N} \models \pi^{2}=\pi^{\prime 2}=\pi^{\prime}=\pi$ and therefore $\mathbf{N} \models \pi=\pi^{2}$, which is impossible if $\pi$ is explicit. So let us suppose that $\pi$ and $\pi^{\prime}$ are both idempotent. A last lemma is needed before we can conclude.

Lemma 3.8. Let $\left(\pi, \pi^{\prime}\right) \in\left(\overline{\mathrm{F}}_{n}(\mathbf{U})\right)^{2}$ be a pseudoidentity satisfied by LI. Suppose that $\pi$ and $\pi^{\prime}$ are idempotent. Then, there exist sequences of explicit operations $\left(r_{m}\right)_{m},\left(s_{m}\right)_{m},\left(t_{m}\right)_{m}$ and $\left(t_{m}^{\prime}\right)_{m}$ such that

$$
\pi=\lim _{m \rightarrow \infty} r_{m} t_{m} s_{m}, \quad \pi^{\prime}=\lim _{m \rightarrow \infty} r_{m} t_{m}^{\prime} s_{m}
$$

and, for every $m \in \mathbb{N}$

$$
\left|r_{m}\right| \geq m, \quad\left|s_{m}\right| \geq m
$$

Proof. Let $\left(u_{m}\right)_{m}$ and $\left(v_{m}\right)_{m}$ be two sequences of explicit operations that converge to $\pi$ and $\pi^{\prime}$ respectively. For any given $k, \mathbf{L I}_{\mathbf{k}}$ satisfies $\pi=\pi^{\prime}$. As $\mathrm{F}_{n}\left(\mathbf{L I}_{\mathbf{k}}\right)$ is finite, it lies in $\mathbf{L I}$; thus, $\mathbf{L I}_{\mathbf{k}}$ satisfies the identity $u_{m}=v_{m}$ for all $m \geq m_{k}$. One can assume for convenience that for these values of $m$, $\left|u_{m}\right|>2 k$ and $\left|v_{m}\right|>2 k$ : otherwise we could first extract a bounded sequence from $\left(\left|u_{m}\right|\right)_{m}$ or $\left(\left|v_{m}\right|\right)_{m}$, and then a constant sequence from $\left(u_{m}\right)_{m}$ or $\left(v_{m}\right)_{m}$. This is impossible since these subsequences converge to idempotent operations and since an idempotent cannot be explicit, by Proposition 3.5.

To say that $\mathbf{L I}_{\mathbf{k}}$ satisfies $u_{m}=v_{m}$ is equivalent to say that $u_{m}$ and $v_{m}$ have the same prefix and same suffix of length $k$. As $\left|u_{m}\right|>2 k$ and $\left|v_{m}\right|>2 k$, we can write $u_{m_{k}}=\tilde{r}_{m_{k}} \tilde{t}_{m_{k}} \tilde{s}_{m_{k}}$ and $v_{m_{k}}=\tilde{r}_{m_{k}} \tilde{t}_{m_{k}}^{\prime} \tilde{s}_{m_{k}}$ with $\left|\tilde{r}_{m_{k}}\right|=\left|\tilde{s}_{m_{k}}\right|=k$. To conclude, take $r_{k}=\tilde{r}_{m_{k}}, s_{k}=\tilde{s}_{m_{k}}, t_{k}=\tilde{t}_{m_{k}}$.

Let us conclude the proof of Theorem 3.1. We may write the implicit operations $\pi$ and $\pi^{\prime}$ as limits of sequences $\left(r_{m} t_{m} s_{m}\right)_{m}$ and $\left(r_{m} t_{m}^{\prime} s_{m}\right)_{m}$ as in Lemma 3.8. By compactness of $\overline{\mathrm{F}}_{n}(\mathbf{U})$, we may assume that $\left(r_{m}\right)_{m},\left(s_{m}\right)_{m},\left(t_{m}\right)_{m}$ and $\left(t_{m}^{\prime}\right)_{m}$ also converge, to $\rho, \sigma, \tau$ and $\tau^{\prime}$ respectively. By Proposition 3.5, $\rho$ and 
$\sigma$ are idempotent since $\lim _{m \rightarrow \infty}\left|r_{m}\right|=\lim _{m \rightarrow \infty}\left|s_{m}\right|=+\infty$. By aperiodicity, U satisfies therefore

$$
\begin{aligned}
\pi & =\rho^{\omega} \tau \sigma^{\omega}=\rho^{\omega}(\rho \tau \sigma) \sigma^{\omega} \\
\pi^{\prime} & =\rho^{\omega} \tau^{\prime} \sigma^{\omega}=\rho^{\omega}\left(\rho \tau^{\prime} \sigma\right) \sigma^{\omega}
\end{aligned}
$$

By hypothesis, $\overline{\mathrm{F}}_{n}(\mathbf{B})$ satisfies $\pi=\pi^{\prime}$ Hence, it satisfies also $\rho \tau \sigma=\rho \tau^{\prime} \sigma$ and it suffices now to use the pseudoidentity (1) to conclude.

\section{Some subpseudovarieties of $\mathrm{LI} \vee \mathrm{B}$}

In this section, we compute the joins $\mathbf{V} \vee \mathbf{W}$ where $\mathbf{V} \in\{\mathbf{L I}, \mathbf{K}, \mathbf{D}, \mathbf{N}\}$ and $\mathbf{W} \in\{\mathbf{B}, \mathbf{S l}\}$.

Theorem 4.1. $\quad$ The pseudovarieties $\mathbf{K} \vee \mathbf{B}$ and $\mathbf{D} \vee \mathbf{B}$ are defined by

$\mathbf{K} \vee \mathbf{B}=\llbracket x^{\omega} y=x^{\omega} y^{2},\left(x^{\omega} y\right)=\left(x^{\omega} y\right)^{2},\left(y x^{\omega}\right)=\left(y x^{\omega}\right)^{2} \rrbracket=\llbracket x^{\omega} y=x^{\omega} y^{2} \rrbracket \cap(\mathbf{B}: \mathbf{N})$

$\mathbf{D} \vee \mathbf{B}=\llbracket y x^{\omega}=y^{2} x^{\omega},\left(x^{\omega} y\right)=\left(x^{\omega} y\right)^{2},\left(y x^{\omega}\right)=\left(y x^{\omega}\right)^{2} \rrbracket=\llbracket y x^{\omega}=y^{2} x^{\omega} \rrbracket \cap(\mathbf{B}: \mathbf{N})$

Proof. We just prove the first of these equalities. The second one is dual. Let $\mathbf{U}$ be the pseudovariety defined by the pseudoidentities

$$
\begin{gathered}
\left(x^{\omega} y\right)=\left(x^{\omega} y\right)^{2}, \quad\left(y x^{\omega}\right)=\left(y x^{\omega}\right)^{2} \\
x^{\omega} y=x^{\omega} y^{2}
\end{gathered}
$$

The containment $\mathbf{K} \vee \mathbf{B} \subseteq \mathbf{U}$ is once again straightforward. We now prove that if $\pi, \pi^{\prime} \in \overline{\mathrm{F}}_{n}(\mathbf{U})$ and $\mathbf{K} \vee \mathbf{B} \models \pi=\pi^{\prime}$, then $\pi=\pi^{\prime}$. We need to prove analogues of Lemma 3.8. We first have:

Lemma 4.2. Let $\pi$ and $\pi^{\prime}$ be two implicit operations of $\overline{\mathrm{F}}_{n}(\mathbf{U})$ that coincide over $\overline{\mathrm{F}}_{n}(\mathbf{B})$. Then, $\mathbf{U}$ satisfies $x^{\omega} \pi=x^{\omega} \pi^{\prime}$.

Proof. As before, we just prove this pseudoidentity for explicit operations, and we deduce the general case by taking the limit. It suffices to show here that $\mathbf{U}$ satisfies $x^{\omega} w w^{\prime} w^{\prime \prime}=x^{\omega} w w^{\prime 2} w^{\prime \prime}$. But $\mathbf{U}$ satisfies

$$
\begin{aligned}
x^{\omega} w w^{\prime} w^{\prime \prime} & =\left(x^{\omega} w\right)^{\omega} w^{\prime} w^{\prime \prime} & \text { as } E\left(\overline{\mathrm{F}}_{n}(\mathbf{U})\right) \text { is an ideal } \\
& =\left(x^{\omega} w\right)^{\omega} w^{\prime 2} w^{\prime \prime} & \text { by }(2) \\
& =x^{\omega} w w^{\prime 2} w^{\prime \prime} &
\end{aligned}
$$

The natural version of Lemma 3.8 is the following:

Lemma 4.3. Let $\pi$ and $\pi^{\prime}$ be idempotent elements of $\overline{\mathrm{F}}_{n}(\mathbf{U})$ such that $\mathbf{K} \models$ $\pi=\pi^{\prime}$. Then, there exist sequences $\left(r_{m}\right)_{m},\left(t_{m}\right)_{m}$ and $\left(t_{m}^{\prime}\right)_{m}$ of explicit operations such that

$$
\pi=\lim _{m \rightarrow \infty} r_{m} t_{m}, \quad \pi^{\prime}=\lim _{m \rightarrow \infty} r_{m} t_{m}^{\prime}
$$

with $\left|r_{m}\right| \geq m$ for every $m \in \mathbb{N}$. 
Proof. It is the same as that of Lemma 3.8, using $\mathbf{K}_{\mathbf{k}}$ instead of $\mathbf{L I}_{\mathbf{k}}$.

In order to complete the proof of Theorem 4.1, we apply the above lemma, and assume furthermore that $\left(r_{m}\right)_{m},\left(t_{m}\right)_{m}$, and $\left(t_{m}^{\prime}\right)_{m}$ converge to $\rho, \tau$ and $\tau^{\prime}$ respectively: we have $\pi=\rho^{\omega} \tau$ and $\pi^{\prime}=\rho^{\omega} \tau^{\prime}$. By Proposition 3.5, $\rho$ is idempotent since $\lim _{m \rightarrow \infty}\left|r_{m}\right|=+\infty$. Therefore, by aperiodicity, $\mathbf{U}$ satisfies

$$
\begin{gathered}
\pi=\rho^{\omega} \tau=\rho^{\omega}(\rho \tau) \\
\pi^{\prime}=\rho^{\omega} \tau^{\prime}=\rho^{\omega}\left(\rho \tau^{\prime}\right)
\end{gathered}
$$

But by hypothesis, $\overline{\mathrm{F}}_{n}(\mathbf{B})$ satisfies $\pi=\pi^{\prime}$, and therefore, it satisfies also $\rho \tau=\rho \tau^{\prime}$. The conclusion follows from Lemma 4.2 .

The next computation is easier:

Theorem 4.4. The pseudovariety $\mathbf{N} \vee \mathbf{B}$ is defined by

$$
\mathbf{N} \vee \mathbf{B}=\llbracket x^{\omega} y=(x y)^{\omega}=x y^{\omega} \rrbracket
$$

Proof. Let $\mathbf{U}=\llbracket x^{\omega} y=(x y)^{\omega}=x y^{\omega} \rrbracket$. It is clear that, if $S \in \mathbf{U}$, then $E(S)$ is an ideal of $S$, that is, $\mathbf{U} \subseteq \mathbf{B} m \mathbf{N}$. Moreover, $\mathbf{N}$ and $\mathbf{B}$ clearly satisfy $x^{\omega} y=(x y)^{\omega}=x y^{\omega}$, so $\mathbf{N} \vee \mathbf{B} \subseteq \mathbf{U}$. Let now $\pi$ and $\pi^{\prime}$ be two implicit operations of $\overline{\mathrm{F}}_{n}(\mathbf{U})$ such that $\mathbf{N} \vee \mathbf{B} \models \pi=\pi^{\prime}$. We need to show that $\pi=\pi^{\prime}$. As in the proof of Theorem 3.1, we verify that if one of $\pi$ and $\pi^{\prime}$ is explicit, then $\mathbf{N}$ satisfies a non trivial identity. Since this is not the case, both $\pi$ and $\pi^{\prime}$ are idempotent. Let $\left(u_{m}\right)_{m}$ and $\left(v_{m}\right)_{m}$ be sequences of explicit operations on $\mathbf{U}$ that converge to $\pi$ and $\pi^{\prime}$ respectively. Since $\overline{\mathrm{F}}_{n}(\mathbf{B})$ is finite, $u_{m} \equiv_{n} v_{m}$ holds for $m$ large enough. Now, U satisfies

$$
\begin{aligned}
\left(w w^{\prime 2} w^{\prime \prime}\right)^{\omega} & =\left(w w^{\prime 2}\right)^{\omega} w^{\prime \prime} \\
& =w\left(w^{\prime 2}\right)^{\omega} w^{\prime \prime} \\
& =w w^{\prime \omega} w^{\prime \prime}
\end{aligned}
$$

and in the same way, $\left(w w^{\prime} w^{\prime \prime}\right)^{\omega}=w w^{\prime \omega} w^{\prime \prime}$. Therefore, $\mathbf{U}$ satisfies $\left(w w^{\prime 2} w^{\prime \prime}\right)^{\omega}=$ $\left(w w^{\prime} w^{\prime \prime}\right)^{\omega}$. Hence, the explicit operations $u_{m}^{\omega}$ and $v_{m}^{\omega}$ are equal for $m$ large enough. Therefore, $\pi=\pi^{\prime}$.

The next result was first proved by Almeida [7]. Recall that Perm is the pseudovariety of all finite semigroups that satisfy a non-trivial permutation identity. It is defined by $x^{\omega} y z x^{\omega}=x^{\omega} z y x^{\omega}$. It is the join of two other pseudovarieties, $\operatorname{Perm}_{(+\infty, 2,0)}=\llbracket x^{\omega} y z=x^{\omega} z y \rrbracket$ and $\operatorname{Perm}_{(\mathbf{0}, \mathbf{2},+\infty)}=\llbracket y z x^{\omega}=z y x^{\omega} \rrbracket$.

Theorem 4.5. Almeida [7] The pseudovariety LI $\vee \mathbf{S l}$ is defined by:

$$
\mathbf{L I} \vee \mathbf{S} \mathbf{l}=\llbracket x^{\omega} y z x^{\omega}=x^{\omega} z y x^{\omega} \rrbracket_{\mathbf{B} \circledast \mathbf{N}}=(\mathbf{B}: \mathbf{N}) \cap \text { Perm }
$$

which can be written as

$$
\mathbf{L I} \vee(\mathbf{B} \cap \mathbf{C o m})=(\mathbf{L I} \vee \mathbf{B}) \cap(\mathbf{L I} \vee \mathbf{C o m})
$$


Proof. The second statement follows from the first and from the fact that LI $\vee$ Com $=$ Perm (Almeida, [2]). There remains to prove the first statement. Let $\mathbf{U}$ be the pseudovariety $(\mathbf{B}(m) \mathbf{N}) \cap$ Perm. It is necessary to know a little more about the structure of $\overline{\mathrm{F}}_{n}(\mathbf{L I} \vee \mathbf{S l})$ to conclude the proof:

Lemma 4.6. Let $\rho, \tau, \tau^{\prime}$ and $\sigma$ be implicit operations of $\overline{\mathrm{F}}_{n}(\mathbf{U})$ such that $c(\rho \tau \sigma)=c\left(\rho \tau^{\prime} \sigma\right)$. Then the pseudovariety $\mathbf{U}$ satisfies $\rho^{\omega} \tau \sigma^{\omega}=\rho^{\omega} \tau^{\prime} \sigma^{\omega}$.

Proof. It suffices to show the lemma for explicit operations $u, v, v^{\prime}$, and $w$ instead of $\rho, \tau, \tau^{\prime}$ and $\sigma$ respectively. The result follows by taking the limit, since the content function is continuous. One can now show that $\mathbf{U}$ satisfies the pseudoidentity (1) and also $x^{\omega} y z t^{\omega}=x^{\omega} z y t^{\omega}$. Now, if two words of $A_{n}^{+}$ have the same content, one can pass from one to the other by using a finite number of times the rewriting rules $x y t \rightarrow x y^{2} t, x y^{2} t \rightarrow x y t$ and $x y z t \rightarrow x z y t$. Therefore, if $r, s \in A_{n}^{+}$have the same content, $\mathbf{U}$ satisfies $x^{\omega} r t^{\omega}=x^{\omega} s t^{\omega}$. So, if $c(u v w)=c\left(u v^{\prime} w\right), \mathbf{U}$ finally satisfies

$$
\begin{aligned}
u^{\omega} v w^{\omega} & =u^{\omega}(u v w) w^{\omega} \\
& =u^{\omega}\left(u v^{\prime} w\right) w^{\omega} \\
& =u^{\omega} v^{\prime} w^{\omega}
\end{aligned}
$$

by aperiodicity since $c(u v w)=c\left(u v^{\prime} w\right)$ by aperiodicity

Now, Theorem 4.5 is a consequence of this lemma: let $\left(\pi, \pi^{\prime}\right) \in \overline{\mathrm{F}}_{n}(\mathbf{U})^{2}$ such that $\mathbf{L I} \vee \mathbf{S l} \models \pi=\pi^{\prime}$. We want to show that $\pi=\pi^{\prime}$. As in the proof of Theorem 3.1, we can assume that both $\pi$ and $\pi^{\prime}$ are idempotent. By Lemma 3.8, $\pi$ and $\pi^{\prime}$ can be written as limits of sequences $\left(r_{m} t_{m} s_{m}\right)_{m}$ and $\left(r_{m} t_{m}^{\prime} s_{m}\right)_{m}$ with $\left|r_{k}\right|=\left|s_{k}\right|=k$. By compactness, we can suppose that $\left(r_{m}\right)_{m},\left(s_{m}\right)_{m},\left(t_{m}\right)_{m}$ and $\left(t_{m}^{\prime}\right)_{m}$ converge to $\rho, \sigma, \tau$ and $\tau^{\prime}$ respectively. Furthermore, by fact 3 of Proposition 3.5, $\rho$ and $\sigma$ have to be idempotent. Therefore, $\pi=\rho^{\omega} \tau \sigma^{\omega}$ and $\pi^{\prime}=\rho^{\omega} \tau^{\prime} \sigma^{\omega}$. Now, Sl satisfies $\pi=\pi^{\prime}$, that is $c(\rho \tau \sigma)=c\left(\rho \tau^{\prime} \sigma\right)$. We then conclude using Lemma 4.6.

With the same techniques, one can compute the pseudovarieties $\mathbf{K} \vee \mathbf{S l}$, $\mathbf{D} \vee \mathbf{S l}$ and $\mathbf{N} \vee \mathbf{S l}$.

Theorem 4.7. $\quad$ The pseudovarieties $\mathbf{K} \vee \mathbf{S l}$ and $\mathbf{D} \vee \mathbf{S l}$ are defined by

$$
\begin{aligned}
& \mathbf{K} \vee \mathbf{S l}=(\mathbf{K} \vee \mathbf{B}) \cap \llbracket x^{\omega} y z=x^{\omega} z y \rrbracket=(\mathbf{K} \vee \mathbf{B}) \cap \operatorname{Perm}_{(+\infty, 2, \mathbf{0})} \\
& \mathbf{D} \vee \mathbf{S l}=(\mathbf{D} \vee \mathbf{B}) \cap \llbracket y z x^{\omega}=z y x^{\omega} \rrbracket=(\mathbf{D} \vee \mathbf{B}) \cap \mathbf{P e r m}_{(\mathbf{0}, \mathbf{2},+\infty)}
\end{aligned}
$$

which can be written as

$$
\begin{aligned}
& \mathbf{K} \vee(\mathbf{B} \cap \mathbf{C o m})=(\mathbf{K} \vee \mathbf{B}) \cap(\mathbf{K} \vee \mathbf{C o m}) \\
& \mathbf{D} \vee(\mathbf{B} \cap \mathbf{C o m})=(\mathbf{D} \vee \mathbf{B}) \cap(\mathbf{D} \vee \mathbf{C o m})
\end{aligned}
$$


Proof. The statements regarding $\mathbf{K}$ and $\mathbf{D}$ are dual, and we prove only that regarding $\mathbf{K}$. The second part of the statement is a consequence of its first part and from the fact that $\mathbf{K} \vee \mathbf{C o m}=\mathbf{P e r m}_{(+\infty, 2, \mathbf{0})}$ (Almeida, [2]). Let again $\mathbf{U}$ be the pseudovariety $(\mathbf{K} \vee \mathbf{B}) \cap \mathbf{P e r m}_{(+\infty, 2, \mathbf{0})}$. The following lemma is analogous to Lemma 4.6:

Lemma 4.8. Let $\rho, \tau$, and $\tau^{\prime}$ be implicit operations of $\overline{\mathrm{F}}_{n}(\mathbf{U})$ such that $c(\rho \tau)=c\left(\rho \tau^{\prime}\right)$. Then the pseudovariety $\mathbf{U}$ satisfies $\rho^{\omega} \tau=\rho^{\omega} \tau^{\prime}$.

Proof. As usual, it suffices to show this lemma for explicit operations $u, v$, and $v^{\prime}$ instead of $\rho, \tau$ and $\tau^{\prime}$ respectively, and the result follows by taking the limit. With the same proof as in Lemma 4.2, one shows that this pseudoidentity holds in $\mathbf{U}$ and that $x^{\omega} y z=x^{\omega} z y$ also holds. It is not difficult to check then, as in Lemma 4.6, that $\mathbf{U}$ satisfies $x^{\omega} u=x^{\omega} u^{\prime}$ if $u$ and $u^{\prime}$ have the same content.

As before, one can then write $\pi=\rho^{\omega} \tau$ and $\pi^{\prime}=\rho^{\omega} \tau^{\prime}$, and it suffices then to use the same technique as for $\mathbf{L I} \vee \mathbf{S I}$.

The last result goes back to Almeida again. Recall that $\mathbf{Z E}$ is the pseudovariety of semigroups in which idempotents are central.

Theorem 4.9. $\quad$ Almeida $[7,3]$ The pseudovariety $\mathbf{N} \vee \mathbf{S l}$ is defined by

$$
\mathbf{N} \vee \mathbf{S l}=(\mathbf{N} \vee \mathbf{B}) \cap \mathbf{Z E}
$$

On can also write

$$
\mathbf{N} \vee(\mathbf{B} \cap \mathbf{C o m})=(\mathbf{N} \vee \mathbf{B}) \cap(\mathbf{N} \vee \mathbf{C o m})
$$

Proof. The inclusion from left to right of each of the equalities is once again clear. Let $\mathbf{U}$ be the pseudovariety $(\mathbf{N} \vee \mathbf{B}) \cap \mathbf{Z E}$. The proof that $\mathbf{U}$ is contained in $\mathbf{N} \vee \mathbf{S l}$ follows the same pattern as the preceding proofs: it suffices to show that U satisfies $\pi=\pi^{\prime}$ if $\pi$ and $\pi^{\prime}$ are two idempotents which have the same content.

The proof of the second equality is easy: the inclusion from left to right is straightforward, and the one from right to left comes from the inclusion $\mathbf{N} \vee$ Com $\subseteq$ $\mathbf{Z E}$. Notice however that $\mathbf{Z E}$ is not permutative, so $\mathbf{Z E} \neq \mathbf{N} \vee \mathbf{C o m}$.

\section{Implicit operations of $\mathrm{LI} \vee \mathrm{B}$}

For comparison, recall that an implicit operation on LI that is not explicit can be viewed as a pair of a left and a right infinite word [7, 34]. Indeed, as $\mathbf{N}$, which does not satisfy any non-trivial identity, is contained in $\mathbf{L I}$, the semigroup $\mathrm{F}_{n}(\mathbf{L I})$ is free over $\left\{x_{1}, \ldots x_{n}\right\}$. If a sequence $\left(u_{p}\right)_{p}$ of $\mathrm{F}_{n}(\mathbf{L I})$ converges in $\overline{\mathrm{F}}_{n}(\mathbf{L I})$, then, this sequence converges in every (finite) semigroup $\mathrm{F}_{n}\left(\mathbf{L I}_{\mathbf{k}}\right) \in \mathbf{L I}$. If it is not a constant sequence, the prefixes (resp. the suffixes) of length $k$ of $u_{p}$ and $u_{q}$ coincide if $p$ and $q$ are large enough. 
Conversely, since the semigroups $\mathrm{F}_{n}\left(\mathbf{L I}_{\mathbf{k}}\right)$ generate $\mathbf{L I}$, every sequence satisfying this condition converges in $\overline{\mathrm{F}}_{n}(\mathbf{L I})$. One can therefore view the non explicit operations as pairs of $A_{n}^{\omega} \times{ }^{\omega} A_{n}$ where ${ }^{\omega} A_{n}$ is the set of left infinite words and $A_{n}^{\omega}$ the set of right infinite words over the alphabet $A_{n}$. The product over $\overline{\mathrm{F}}_{n}(\mathbf{L I})$ is defined by

$$
\begin{aligned}
u \cdot(\pi, \rho) & =(u \pi, \rho) & & \text { if } \pi \in A_{n}^{\omega}, \rho \in{ }^{\omega} A_{n} \text { and } u \in A_{n}^{+} \\
(\pi, \rho) \cdot u & =(\pi, \rho u) & & \text { if } \pi \in A_{n}^{\omega}, \rho \in{ }^{\omega} A_{n} \text { and } u \in A_{n}^{+} \\
(\pi, \rho) \cdot\left(\pi^{\prime}, \rho^{\prime}\right) & =\left(\pi, \rho^{\prime}\right) & & \text { if } \pi, \pi^{\prime} \in A_{n}^{\omega} \text { and } \rho, \rho^{\prime} \in{ }^{\omega} A_{n}
\end{aligned}
$$

In other words, $\overline{\mathrm{F}}_{n}(\mathbf{L I}) \backslash \mathrm{F}_{n}(\mathbf{L I})$ is a rectangular band and $\overline{\mathrm{F}}_{n}(\mathbf{L I})$ is a nilpotent extension of a rectangular band.

The semigroup $\overline{\mathrm{F}}_{n}(\mathbf{L I} \vee \mathbf{B})$ has quite a similar structure: since $\mathbf{L I} \vee \mathbf{B}$ contains $\mathbf{N}$, the semigroup $\mathrm{F}_{n}(\mathbf{L I} \vee \mathbf{B})$ is the free semigroup over $\left\{x_{1}, \ldots x_{n}\right\}$ (equipped with the discrete topology). Furthermore, as an implicit operation $\pi \in \overline{\mathrm{F}}_{n}(\mathbf{B} \mathrm{m} \mathbf{\mathrm { N }})$ is determined by its value over $\mathbf{L I}$ on one hand and by its value over $\overline{\mathrm{F}}_{n}(\mathbf{B})$ on the other hand, a non explicit operation over $\mathbf{L I} \vee \mathbf{B}$ can be viewed as a triple of $A_{n}^{\omega} \times \mathrm{F}_{n}(\mathbf{B}) \times{ }^{\omega} A_{n}$. The product over $\overline{\mathrm{F}}_{n}(\mathbf{L I})$ is now defined by

$$
\begin{array}{ll}
v \cdot(\pi, u, \rho)=(v \pi, v u, \rho) & \text { if } \pi \in A_{n}^{\omega}, \rho \in{ }^{\omega} A_{n} \text { and } v \in A_{n}^{+} \text {and } u \in \mathrm{F}_{n}(\mathbf{B}) \\
(\pi, u, \rho) \cdot v=(\pi, u v, \rho v) & \text { if } \pi \in A_{n}^{\omega}, \rho \in{ }^{\omega} A_{n} \text { and } v \in A_{n}^{+} \text {and } u \in \mathrm{F}_{n}(\mathbf{B}) \\
(\pi, u, \rho) \cdot\left(\pi^{\prime}, u^{\prime}, \rho^{\prime}\right)=\left(\pi, u u^{\prime}, \rho^{\prime}\right) & \text { if } \pi, \pi^{\prime} \in A_{n}^{\omega}, \rho, \rho^{\prime} \in{ }^{\omega} A_{n}, u, u^{\prime} \in \mathrm{F}_{n}(\mathbf{B})
\end{array}
$$

where the product of $u$ by $v$ is evaluated in free band.

In other words, non explicit operations on $\mathbf{L I} \vee \mathbf{B}$ form a band, more precisely the direct product of the rectangular band $A_{n}^{\omega} \times{ }^{\omega} A_{n}$ by the free band. Therefore, the semigroup $\overline{\mathrm{F}}_{n}(\mathbf{L I} \vee \mathbf{B})$ is a nilpotent extension of a band, which is not surprising since $\mathbf{L I} \vee \mathbf{B}=\mathbf{B} \rightarrow \mathbf{N}$.

\section{Acknowledgments}

Many thanks are due to Jean-Éric Pin for his help and comments. The question that motivates this paper was asked by Jorge Almeida. I am grateful for his remarks and encouragements. I also thank Pascal Weil for his helpful advices and the for attention he paid for this work. Finally, I would like to thank M. Volkov for pointing out a mistake in the first version of the paper and providing a proof for Lemma 3.7.

\section{References}

[1] D. Albert, R. Baldinger, and J. Rhodes, Undecidability of the identity problem for finite semigroups, J. Symbolic Logic 57 (1992), no. 1, 179-192.

[2] J. Almeida, Power pseudovarieties of semigroups I, Semigroup Forum 33 (1986), 357-373.

[3] _ Some pseudovariety joins involving the pseudovariety of finite groups, Semigroup Forum 37 (1988), 53-57. 
[4] $16-32$.

[5] _ Equations for pseudovarieties, Formal properties of Finite Automata and Applications (J.-E. Pin, ed.), Lect. Notes in Comp. Sci., vol. 386, Springer, 1989, pp. 148-164.

[6] _ On the membership problem for pseudovarieties of commutative semigroups, Semigroup Forum 42 (1991), 47-51.

[7] _ Finite semigroups and universal algebra, Series in Algebra, vol. 3, World Scientific, 1995.

[8] J. Almeida and A. Azevedo, The join of the pseudovarieties of $\mathcal{R}$-trivial and $\mathcal{L}$-trivial monoids, J. Pure and Applied Algebra 60 (1989), 129-137.

[9] _ On regular implicit operations, Portugaliæ Matemática 50 (1993), no. $1,35-61$.

[10] J. Almeida and P. Weil, Reduced factorizations in free profinite groups and join decomposition of pseudovarieties, Int. J. Algebra and Computation 4 (1994), 375-403.

[11] _ Relatively free profinite monoids: an introduction and examples, NATO Advanced Study Institute, Semigroups, Formal Languages and Groups (J.B. Fountain and V.A.R. Gould, eds.), Kluwer, 1995, pp. 73-117.

[12] A. Azevedo, Operations preserving homomorphisms on the class of finite semigroups DS, Actas $2^{\circ}$ Encontro de Algebristas Portugueses (Porto), 1987, pp. 33-43.

[13] — Operações impl'ıcitas sobre pseudovariedades de semigrupos. aplicações, Doctoral dissertation, Universidade do Porto, 1989.

[14] A. Azevedo, The join of the pseudovariety $\boldsymbol{J}$ with permutative pseudovarieties, Lattices, Semigroups and Universal Algebra (J. Almeida et al., ed.), Plenum, 1990.

[15] A.P. Biryukov, Varieties of idempotent semigroups, Algebra Logika 9 (1970), 255-273.

[16] N. Bourbaki, Éléments de mathématiques, topologie générale, vol. I-IV, Hermann, Paris, 1971.

[17] S. Burris and H.P. Sankappanavar, A course in universal algebra, Springer, Berlin, 1981.

[18] C. Fennemore, All varieties of bands, Semigroup Forum 1 (1970), 172-179.

[19] _ All varieties of bands, Math. Nachr. 48 (1971), 237-262. 
[20] J.A. Gerhard, The lattice of equational classes of idempotents semigroups, J. Algebra 15 (1970), 195-224.

[21] _ Semigroups with an idempotent power I, Semigroup Forum 14 (1977), $137-141$.

[22] J.M. Howie, An introduction to semigroup theory, Academic Press, London, 1976.

[23] O.G. Kharlampovich and M. Sapir, Algorithmic problems in varieties, to appear.

[24] M. Lothaire, Combinatorics on words, Encyclopedia of Mathematics, vol. 17, Addison Wesley, Reading, MA, 1983.

[25] J.E. Pin, Varieties of formal languages, Plenum, London, 1986.

[26] L. Polák, On varieties of completely regular semigroups I, Semigroup Forum 32 (1985), 97-123.

[27] _ On varieties of completely regular semigroups II, Semigroup Forum 36 (1987), 73-88.

[28] _ On varieties of completely regular semigroups III, Semigroup Forum 37 (1988), 1-30.

[29] J. Reiterman, The Birkhoff theorem for finite algebras, Algebra Universalis 14 (1982), 1-10.

[30] J. Rhodes, New techniques in global semigroup theory, Proc. Chico Conf. Semigroups and their applications (S. Goberstein and P. Higgins, eds.), D. Reidel, 1987, pp. 25-35.

[31] Eilenberg S., Automata, languages and machines, vol. A, B, Academic Press, New York, 1974-1976.

[32] P.G. Trotter and M.V. Volkov, The finite basis problem in the pseudovariety joins of aperiodic semigroups with groups, Semigroup Forum 52 (1996), 83-91.

[33] M.V. Volkov, On a class of semigroup pseudovarieties without finite pseudoidentity basis, Int. J. Algebra and Computation 5 (1995), 127-135.

[34] P. Weil, Implicit operations on pseudovarieties: an introduction, Semigroups and Monoids and Applications (J. Rhodes, ed.), World Scientific, 1991.

[35] M. Zeitoun, A simple example of a non-finitely based semigroup variety, In preparation. 